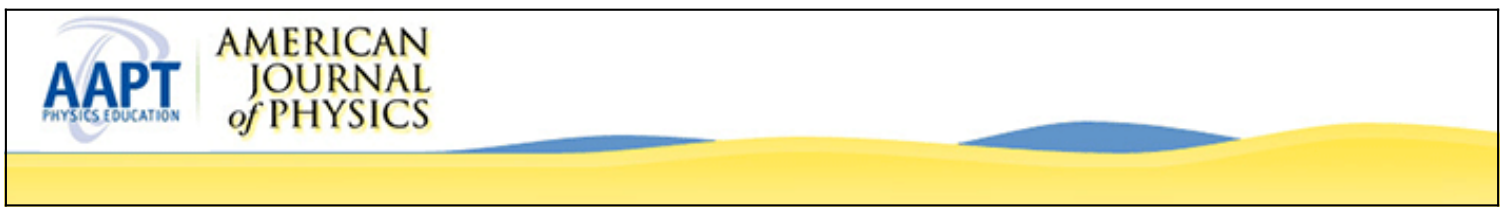

\title{
Linear least-squares fits with errors in both coordinates. II: Comments on parameter variances
}

B. Cameron Reed

Citation: American Journal of Physics 60, 59 (1992); doi: 10.1119/1.17044

View online: http://dx.doi.org/10.1119/1.17044

View Table of Contents: http://scitation.aip.org/content/aapt/journal/ajp/60/1?ver=pdfcov

Published by the American Association of Physics Teachers

\section{Articles you may be interested in}

Comment on "Linear least-squares fits with errors in both coordinates," by B. C. Reed [Am. J. Phys. 57 , 642-646 (1989)]

Am. J. Phys. 59, 472 (1991); 10.1119/1.16826

Comment on "Linear least-squares fits with errors in both coordinates," by B. C. Reed [Am. J. Phys. 57 , 642-646 (1989)]

Am. J. Phys. 58, 1209 (1990); 10.1119/1.16204

Erratum: "Linear least-squares fits with errors in both coordinates" [Am. J. Phys. 5 7, 642-646 (1989)]

Am. J. Phys. 58, 189 (1990); 10.1119/1.16506

Error analysis for parameters determined in nonlinear least-squares fits

Am. J. Phys. 58, 160 (1990); 10.1119/1.16228

Linear least-squares fits with errors in both coordinates

Am. J. Phys. 57, 642 (1989); 10.1119/1.15963

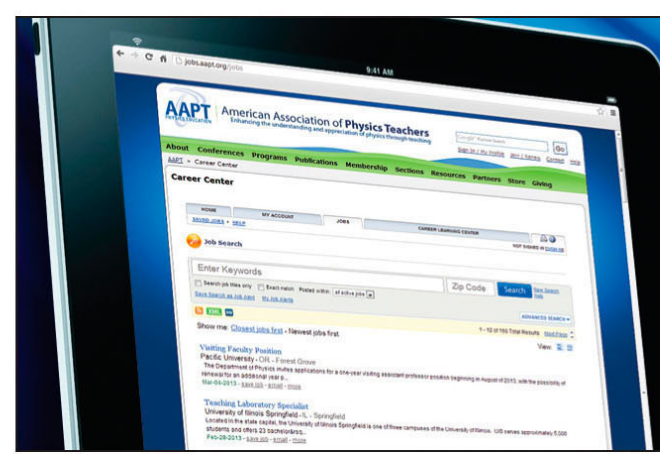

American Association of Physics Teachers

Explore the AAPT Career Center access hundreds of physics education and other STEM teaching jobs at two-year and four-year colleges and universities.

\section{http://jobs.aapt.org}




\title{
Linear least-squares fits with errors in both coordinates. II: Comments on parameter variances
}

\author{
B. Cameron Reed \\ Department of Physics, Saint Mary's University, Halifax, Nova Scotia B3H 3C3, Canada
}

(Received 10 December 1990; accepted 18 April 1991)

Corrected expressions for parameter variances in linear (straight-line) least-squares fits given in an earlier paper [B. C. Reed, Am. J. Phys. 57, 642-646 (1989)] are presented and discussed. In addition, some simplifications to the solution given in that paper are presented, and some comments made as to the role of weighting.

\section{INTRODUCTION}

In the July 1989 edition of this Journal, this author published a paper concerning linear least-squares fits (that is, straight-line fits) in cases where there are errors in both coordinates $^{1}$ (hereafter Paper I). This paper drew attention to various numerical problems that can arise on attempting to implement the iterative solution to this problem originally put forth by York, ${ }^{2}$ showed how one can circumvent these problems in practice, and made available a simple program for doing the computations on a PC. In addition, it was shown that York's solution is exact, and immune from problems encountered in attempts to modify conventional least-squares algorithms. ${ }^{3-6}$ Since publication of this paper, many interested parties have written to the author with detailed accounts of their experiences with a variety of algorithms claiming to address this problem. These experiences are clearly converging on two conclusions: (i) the various algorithms agree as to the correct values of the slope $m$ and intercept $c$, and (ii) there is considerable disagreement as to the variances $\sigma_{m}$ and $\sigma_{c}$ of the slope and intercept that various methods report. Unfortunately, the expressions quoted for $\sigma_{m}$ and $\sigma_{c}$ in Paper I have proven to be erroneous.

Given that users who have developed programs based on Paper I may not be aware of this shortcoming, a discussion giving a corrected treatment is needed. This is the main purpose of the present paper. However, this paper is also much more than an erratum, for a number of sundry issues are also addressed. Specifically, a simplification of the "least-squares cubic" is discussed, attention is drawn to some relevant references the author was not aware of at the time of writing Paper I, results for a commonly used example are discussed in detail, and some remarks on the role of weighting are made. Since the literature on this problem goes back over a century, the present paper will surely not be the last word on it; the purpose here is therefore not so much to introduce new results as to clarify old ones and to provide detailed examples against which others can check their own algorithms.

The outline of this paper is as follows. In Sec. II York's solution to the best-fit line is summarized and simplified. In Sec. III the difficulty with York's expression for $\sigma_{m}$ is examined, and a corrected expression offered. Detailed results for a well-known example ("Pearson's data with York's weights") are presented, along with revised results for examples I and II in Paper I. Section IV discusses some sundry points. Explicit expressions for $\sigma_{i n}$ and $\sigma_{c}$ appear in the Appendix.

\section{YORK'S SOLUTION}

Assume that one has a set of $N$ data-point pairs $\left(x_{i}, y_{i}\right)$; each point has its own weighting factors in $x$ and $y$, designated as $W\left(x_{i}\right)$ and $W\left(y_{i}\right)$, which are conventionally assigned as the inverses of the squares of the measurement uncertainties (see Sec. IV). The problem is to find the values of the parameters $m$ and $c$ that yield the "best-fit" of the model equation

$$
Y=m X+c
$$

to the data, where $X$ and $Y$ are the predicted or calculated values of the data points, that is, their values after adjustment to the best-fit line. "Best-fit" is defined as minimizing the sum of the weighted squared residuals, commonly designated as $\mathbf{S}$ :

$$
\mathbf{S}=\sum_{i=1}^{N}\left[W\left(x_{i}\right)\left(x_{i}-X_{i}\right)^{2}+W\left(y_{i}\right)\left(y_{i}-Y_{i}\right)^{2}\right] .
$$

[Note: In most textbook treatments, $(X, Y)$ designates an observed point, and $(x, y)$ the predicted. We reverse this notation to remain consistent with Paper I]. York ${ }^{2}$ showed that the best-fit slope is given by solving the equation

$$
f(m)=m^{3}-3 \alpha m^{2}+3 \beta m-\gamma=0,
$$

where

$$
\begin{aligned}
& \alpha=\left(2 \sum \frac{W_{i}^{2} U_{i} V_{i}}{W\left(x_{i}\right)}\right)(3 \delta)^{-1}, \\
& \beta=\left(\sum \frac{W_{i}^{2} V_{i}^{2}}{W\left(x_{i}\right)}-\sum W_{i} U_{i}^{2}\right)(3 \delta)^{-1}, \\
& \gamma=\left(-\sum W_{i} U_{i} V_{i}\right) \delta^{-1},
\end{aligned}
$$

with

$$
\delta=\sum \frac{W_{i}^{2} U_{i}^{2}}{W\left(x_{i}\right)}
$$

and where the "overall" or "effective" weight $W_{i}$ for a given point is given by

$$
W_{i}=\frac{W\left(x_{i}\right) W\left(y_{i}\right)}{m^{2} W\left(y_{i}\right)+W\left(x_{i}\right)} .
$$

In Eqs. (4)-(7), $U_{i}$ and $V_{i}$ are defined by

$$
\begin{aligned}
& U_{i}=x_{i}-\langle x\rangle, \\
& V_{i}=y_{i}-\langle y\rangle,
\end{aligned}
$$

where $\langle x\rangle$ and $\langle y\rangle$ are given by 


$$
\langle x\rangle=\sum W_{i} x_{i}\left(\sum W_{i}\right)^{-1}
$$

and

$$
\langle y\rangle=\sum W_{i} y_{i}\left(\sum W_{i}\right)^{-1},
$$

with all sums in Eqs. (4)-(12) running from 1 to $N$.

The central problem in finding the best-fit slope is then to find that root of Eq. (3) that minimizes S. York derived Eq. (3) by applying the method of undetermined multipliers to Eq. (1) and demanding that $\mathbf{S}$ be minimized; he termed Eq. (3) the "least-squares cubic" because it appears to have the form of a cubic equation in $m$. However, this is illusory as the "coefficients" $\alpha, \beta$, and $\gamma$, are in fact all functions of $m$ through the $U_{i}, V_{i}, W_{i}$, and $\delta$. As discussed in Paper I, the simplest approach is to plot $f(m)$ vs $m$ and examine the zeros.

Once $m$ is known, the intercept $c$ is given by

$$
c=\langle y\rangle-m\langle x\rangle \text {, }
$$

and the value of $\mathbf{S}$ can be expressed as

$\mathbf{S}=\sum W_{i}\left(y_{i}-c-m x_{i}\right)^{2}=\sum W_{i}\left(V_{i}-m U_{i}\right)^{2}$.

York gives (erroneously) the variances in the slope and intercept as

$$
\sigma_{m}^{2}=\frac{\mathbf{S}}{N-2}\left(\sum W_{i} U_{i}^{2}\right)^{-1}
$$

and

$$
\sigma_{c}^{2}=\left[\sum W_{i} x_{i}^{2}\left(\sum W_{i}\right)^{-1}\right] \sigma_{m}^{2} .
$$

Before examining the difficulties with these last two equations, we give a simpler formulation ${ }^{7}$ of $\mathrm{Eq}$. (3). Cancelling factors of 3 and clearing the factor of $\delta$ in the denominator of Eq. (3) yields, after some slight rearrangement

$$
\begin{aligned}
& m^{3}\left(\sum \frac{W_{i}^{2} U_{i}^{2}}{W\left(x_{i}\right)}\right)-2 m^{2}\left(\sum \frac{W_{i}^{2} U_{i} V_{i}}{W\left(x_{i}\right)}\right)+m\left(\sum \frac{W_{i}^{2} V_{i}^{2}}{W\left(x_{i}\right)}\right) \\
& \quad=m\left(\sum W_{i} U_{i}^{2}\right)-\left(\sum W_{i} U_{i} V_{i}\right) .
\end{aligned}
$$

Now, Eq. (8) can be expressed as

$$
\left(\frac{m^{2}}{W\left(x_{i}\right)}+\frac{1}{W\left(y_{i}\right)}\right) W_{i}=1 \text {. }
$$

If Eq. (18) is inserted within the sums of the two terms on the right side of Eq. (17) (equivalent to multiplying through by 1 ), one finds after a little algebra that the "leastsquares cubic" can be expressed, with no loss of generality, as a "least-squares quadratic":

$$
\begin{aligned}
& m^{2}\left(\sum \frac{W_{i}^{2} U_{i} V_{i}}{W\left(x_{i}\right)}\right)+m \sum W_{i}^{2}\left(\frac{U_{i}^{2}}{W\left(y_{i}\right)}-\frac{V_{i}^{2}}{W\left(x_{i}\right)}\right) \\
& \quad-\left(\sum \frac{W_{i}^{2} U_{i} V_{i}}{W\left(y_{i}\right)}\right)=0,
\end{aligned}
$$

which we write for convenience as

$$
g(m)=A m^{2}+B m-C=0 \text {. }
$$

Do not confuse the coefficient $C$ defined in Eqs. (19) and (20) with the best-fit intercept $c$. Williamson, ${ }^{8}$ who also investigated (and corrected) problems with York's param- eter-variance expressions, has shown that the equation for the best-fit slope can actually be cast into a pseudo-linear form. In the present paper, however, I will stick to the form of Eq. (20) to effect a completely independent analysis from that of Williamson.

\section{PARAMETER VARIANCES}

A proper treatment of error propagation ${ }^{9,10}$ shows that the variance in the slope of the best-fit line should be computed via

$$
\sigma_{m}^{2}=\frac{\mathbf{S}}{N-2} \sum_{j}\left[\frac{1}{W\left(y_{j}\right)}\left(\frac{\partial m}{\partial y_{j}}\right)^{2}+\frac{1}{W\left(x_{j}\right)}\left(\frac{\partial m}{\partial x_{j}}\right)^{2}\right],
$$

with a similar expression holding for $\sigma_{c}^{2}$ but with derivatives of $c$ instead of $m$. In writing Eq. (21), we have assumed that weights are assigned in the usual inverse-square fashion: $W\left(x_{i}\right)=1 / \sigma^{2}\left(x_{i}\right)$ and $W\left(y_{i}\right)=1 / \sigma^{2}\left(y_{i}\right)$.

The factor of $S /(N-2)$ (the "external standard deviation") appearing in Eq. (21) deserves some comment. Some authors (Williamson) do not include this factor, although the majority of statistical authorities do so. An intuitive justification for its inclusion can be given as follows. Suppose that one optimistically assigns each of the $W\left(x_{i}\right)$ and $W\left(y_{i}\right)$ to be ten times greater than their true values. The derived slope, intercept, residuals, and values of $\left(\partial m / \partial x_{j}\right)$ and $\left(\partial m / \partial y_{j}\right)$ will all be unchanged, but the sum over $j$ in Eq. (21) would come out ten times too small. On the intuitive belief that $\sigma_{m}$ should remain invariant under such a scaling (after all, the relative weights of each point are unchanged), it becomes necessary to multiply by a factor (such as $\mathbf{S}$ ) directly proportional to the weights. A further aspect of Eq. (21) is that inasmuch as it incorporates only the lowest-order terms in a Taylor-series expansion, it cannot be said to yield "exact" values of the parameter variances, contrary to what Williamson claims in his paper. [The values of $m$ and $c$ derived from Eqs. (3) or (20) are, however, exact in that no approximations have been made in their derivations].

The derivatives appearing in Eq. (21) are straightforward if tedious to compute. One proceeds by differentiating Eq. (20) with respect to $x_{j}$ :

$$
(2 m A+B)\left(\frac{\partial m}{\partial x_{j}}\right)+m^{2}\left(\frac{\partial A}{\partial x_{j}}\right)+m\left(\frac{\partial B}{\partial x_{j}}\right)-\left(\frac{\partial C}{\partial x_{j}}\right)=0,
$$

and solving for $\left(\partial m / \partial x_{j}\right)$. Since $A, B$, and $C$ are also all functions of $m$ the result is a tedious calculation involving extensive application of the chain rule. The results are given explicitly in Appendix A, where it can be seen that they are considerably more complex than York's approximate expression, Eq. (15).

York appears to have derived Eq. (15) from Deming's treatment of this problem. ${ }^{9}$ While it is difficult to say precisely from where York's expression derives, an informed speculation can be made from the form of his result. Comparing Eqs. (15) and (21) indicates that York has taken

$$
\sum_{j}\left[\frac{1}{W\left(y_{j}\right)}\left(\frac{\partial m}{\partial y_{j}}\right)^{2}+\frac{1}{W\left(x_{j}\right)}\left(\frac{\partial m}{\partial x_{j}}\right)^{2}\right] \approx\left(\sum_{j} W_{j} U_{j}^{2}\right)^{-1} .
$$

The sum on the right side of this result turn up in York's work in the case where the $x_{i}$ are not subject to error. In this case $W_{i}=W\left(y_{i}\right)\left[\mathrm{Eq}\right.$. (8) with $W\left(x_{i}\right) \rightarrow \infty$; the 
$\left(\partial m / \partial x_{j}\right)$ term in Eq. (21) vanishes], and his leastsquares cubic reduces to

$$
m\left(\sum W\left(y_{i}\right) U_{i}^{2}\right)-\left(\sum W\left(y_{i}\right) U_{i} V_{i}\right)=0 .
$$

York's expression for $\sigma_{m}^{2}$ then follows if one adopts the approximation $W\left(y_{i}\right) / \Sigma W\left(y_{i}\right) \approx 0$. Whatever the detail of its derivation, York's result appears to involve some inappropriate approximations, and discontinuance of its use in favor in Eq. (21) is urged forthwith.

\section{A. Observed versus calculated points}

Another aspect to this problem not often discussed is that some statistical authorities prefer to evaluate the derivatives in Eq. (21) at the calculated (as opposed to observed) values of the data points. ${ }^{11}$ This is because the Taylor-series used to calculate $\sigma_{m}$ is actually expanded about the calculated points; see, in particular, Sec. 53 of Deming. ${ }^{9}$ In practical circumstances this difference is a small one for well-correlated data, as the examples below indicate. In terms of the observed points $\left(x_{i}, y_{i}\right)$ the calculated points $\left(X_{i}, Y_{i}\right)$ were shown by York to be given by

$$
X_{i}=x_{i}-\lambda_{i} m / W\left(x_{i}\right)
$$

and

$$
Y_{i}=y_{i}+\lambda_{i} / W\left(y_{i}\right),
$$

where the Lagrangian multipliers $\lambda_{i}$ are given by

$$
\lambda_{i}=W_{i}\left(c+m x_{i}-y_{i}\right) .
$$

It is worthwhile to point out that if one takes this approach, then all of $A, B, C, U_{i}, V_{i}$, etc., must be reevaluated at the calculated points after the best-fit slope has been determined, before proceeding with the computation of $\sigma_{m}$ and $\sigma_{c}$.

\section{B. Examples}

As a guide for readers debugging their own programs, I give here some examples. The first is a case commonly seen in the literature , $^{5,12}$ - "Pearson's data with York's weights." The original data traces back to a paper by Karl Pearson in $19011^{14}$ the weights are from York's 1966 paper. The data are given in Table I. The best-fit slope and intercept are found to be $(m, c)=(-0.4805,5.4799)$; the sum of weighted squared residuals is $\mathbf{S}=11.866$. These figures are in agreement with those quoted in Refs. 5, 12, and 13. Equation (21), and its counterpart for $\sigma_{c}$ with derivatives evaluated at the observed points, ${ }^{15}$ gives $\left(\sigma_{m}, \sigma_{c}\right)$

Table I. Pearson's data with York's weights.

\begin{tabular}{rcccc}
\hline$i$ & $x_{i}$ & $y_{i}$ & $W\left(x_{i}\right)$ & $W\left(y_{i}\right)$ \\
\hline 1 & 0.0 & 5.9 & 1000 & 1 \\
2 & 0.9 & 5.4 & 1000 & 1.8 \\
3 & 1.8 & 4.4 & 500 & 4 \\
4 & 2.6 & 4.6 & 800 & 8 \\
5 & 3.3 & 3.5 & 200 & 20 \\
6 & 4.4 & 3.7 & 80 & 20 \\
7 & 5.2 & 2.8 & 60 & 70 \\
8 & 6.1 & 2.8 & 20 & 70 \\
9 & 6.5 & 2.4 & 1.8 & 100 \\
10 & 7.4 & 1.5 & 1 & 500 \\
\hline
\end{tabular}

$=(0.0702,0.3555)$. Evaluating at the calculated points gives only very slightly different results: $\left(\sigma_{m}, \sigma_{c}\right)=(0.0706,0.3592)$.

For the data of example $\mathbf{I}$ in Paper $\mathrm{I},(m, c)$ $=(1.1668,-0.3652), \quad \mathbf{S}=578.05, \quad\left(\sigma_{m}, \sigma_{c}\right)_{\text {obs }}$ $=(0.1704,0.1561)$, and $\left(\sigma_{m}, \sigma_{c}\right)_{\text {calc }}=(0.1470,0.1348)$. For the data example II in Paper I $(m, c)=(4.5437,-17.484), \quad\left(\sigma_{m}, \sigma_{c}\right)_{\mathrm{obs}}=(14.476$, 72.898), $\left(\sigma_{m}, \sigma_{c}\right)_{\mathrm{calc}}=(7.0432,35.551)$, and $\mathbf{S}=13.956$. In this latter case a significant difference appears between the parameter variances computed using the observed versus calculated points; this is because the data were produced by a random number generator and are poorly correlated. The author's experience is that $\sigma_{\text {calc }}$ is generally less than $\sigma_{\text {obs }}$.

For all three of these examples, Chong ${ }^{16}$ has verified independently that these results (with derivatives evaluated at the observed points) are in exact agreement with those predicted by Williamson's expressions, and Jefferys ${ }^{17}$ has confirmed that the results obtained for example I are in agreement with his own software, which utilizes the calculated values of the data points.

\section{SUNDRY REMARKS}

The most important lesson from these results is that when one quotes parameter variances for a least-squares fit, define exactly how the variances were obtained.

A revised FORTRAN program to find the best-fit $m$ via the roots of the "least-squares quadratic," and generate corresponding estimates for $\sigma_{m}$ and $\sigma_{c}$ based on Eq. (21) using both observed and calculated coordinates is available from the author. Including extensive comments, the program runs to about 315 lines. A challenging project for anyone with a lot of patience or a good symbolic manipulator would be to compute the second-order contributions to $\sigma_{m}$ and $\sigma_{c}$.

To close, it seems worthwhile to make a few remarks on the role of weighting. Standard practice is to assign weights as the inverse squares of the measurement uncertainties; this is based fundamentally on the assumption of normally distributed measurement errors; ${ }^{9,10}$ in fact, Deming defines weights in this way in his Sec. 11. A more intuitively appealing argument for this approach can be given as follows. Imagine a situation where the data are such that $0 \leqslant x \leqslant 1,0 \leqslant y \leqslant 10, \sigma\left(x_{i}\right) \approx 0.1$, and $\sigma\left(y_{i}\right) \approx 1$. In both dimensions the (uncertainty/data) ratio is typically 0.1 , but if weights are assigned in the usual way, then we would have $W\left(x_{i}\right) \approx 100$ vs $W\left(y_{i}\right) \approx 1$, which appears to "unfairly" favor the $x$ direction. To preserve the sense that $x$ and $y$ should contribute equally to the solution, one might be tempted to divide all of the $y_{i}$ and $\sigma\left(y_{i}\right)$ by 10 to produce equal weights in each dimension. But if one changes the weights, will not the best-fit slope change? The answer to this can be obtained by working through the effect of such a scaling in the least-squares quadratic: only when weights are assigned as inverse-squares of the errors will the scaled best-fit slope come out to be exactly one-tenth of the unscaled slope. Thus if weights are assigned as the inverse squares of the errors, one need never invoke any scaling; $x$ and $y$ will contribute to the solution "as they should."

\section{ACKNOWLEDGMENTS}

It is a great pleasure to thank all of those individuals who wrote to me concerning paper I and who stimulated me to 
examine this problem further. In particular, Dr. Delano Chong, Dr. Bill Jefferys, and Dr. Matthew Lybanon shared a number of results and valuable comments. This work was supported by Natural Sciences and Engineering Research Council of Canada operating Grant A8580.

\section{APPENDIX: EXPRESSIONS FOR PARAMETER DERIVATIVES}

Here, we give explicit expressions for the derivatives appearing in Eq. (21) and its counterpart for $\sigma_{c}^{2}$. After many hours of algebra, one finds

$$
\begin{aligned}
& \frac{\partial m}{\partial x_{j}}=-\frac{\left(m^{2} D D_{j}+m E E_{j}-F F_{j}\right)}{\left(2 m A+B-A A m^{2}+B B m-C C\right)}, \\
& \frac{\partial m}{\partial y_{j}}=-\frac{\left(m^{2} G G_{j}-2 m D D_{j}-E E_{j} / 2\right)}{\left(2 m A+B-A A m^{2}+B B m-C C\right)} .
\end{aligned}
$$

$A$ and $B$ are defined in Eqs. (19) and (20). The other factors are defined by

$$
\begin{aligned}
A A= & 4 m \sum \frac{W_{i}^{3} U_{i} V_{i}}{W^{2}\left(x_{i}\right)}-\frac{W(H H)(J J)}{m} \\
B B= & -\sum W_{i}^{2}\left[4 m \frac{W_{i}}{W\left(x_{i}\right)}\left(\frac{U_{i}^{2}}{W\left(y_{i}\right)}-\frac{V_{i}^{2}}{W\left(x_{i}\right)}\right)\right. \\
& \left.-\frac{2 V_{i}(H H)}{W\left(x_{i}\right)}+\frac{2 U_{i}(J J)}{W\left(y_{i}\right)}\right] \\
C C= & -\sum \frac{W_{i}^{2}}{W\left(y_{i}\right)}\left[4 m \frac{W_{i} U_{i} V_{i}}{W\left(x_{i}\right)}\right. \\
& \left.+V_{i}(J J)+U_{i}(H H)\right] \\
D D_{j}= & \sum \frac{W_{i}^{2} V_{i}}{W\left(x_{i}\right)}\left(\delta_{i j}-\frac{W_{j}}{W}\right) \\
E E_{j}= & 2 \sum \frac{W_{i}^{2} U_{i}}{W\left(y_{i}\right)}\left(\delta_{i j}-\frac{W_{j}}{W}\right) \\
F F_{j}= & \sum \frac{W_{i}^{2} V_{i}}{W\left(y_{i}\right)}\left(\delta_{i j}-\frac{W_{j}}{W}\right),
\end{aligned}
$$

and

$$
G G_{j}=\sum \frac{W_{i}^{2} U_{i}}{W\left(x_{i}\right)}\left(\delta_{i j}-\frac{W_{j}}{W}\right)
$$

where

$$
\begin{aligned}
& W=\sum W_{i}, \\
& H H=-\frac{2 m}{W} \sum \frac{W_{i}^{2} V_{i}}{W\left(x_{i}\right)},
\end{aligned}
$$

$$
J J=-\frac{2 m}{W} \sum \frac{W_{i}^{2} U_{i}}{W\left(x_{i}\right)},
$$

and where $\delta_{i j}$ is the usual Kronecker delta. All sums are over $i=1$ to $N$. Expressions for the derivatives of the intercept $c$ with respect to $x_{j}$ and $y_{j}$ follow from the above and Eqs. (11)-(13):

$$
\begin{aligned}
& \frac{\partial c}{\partial x_{j}}=[(H H)-m(J J)-\langle x\rangle]\left(\frac{\partial m}{\partial x_{j}}\right)-\frac{m W_{j}}{W}, \\
& \frac{\partial c}{\partial y_{j}}=[(H H)-m(J J)-\langle x\rangle]\left(\frac{\partial m}{\partial y_{j}}\right)+\frac{W_{j}}{W} .
\end{aligned}
$$

'B. C. Reed, "Linear least-squares fits with errors in both coordinates," Am. J. Phys. 57, 642-646 (1989); Erratum in Am. J. Phys. 58, 189 (1990).

D. York, "Least-squares fitting of a straight line," Can. J. Phys. 44, 1079-1086 (1966)

${ }^{3}$ D. R. Barker and L. M. Diana, "Simple method for fitting data when both variables have uncertainties," Am. J. Phys. 42, 224-227 (1974).

${ }^{4} \mathrm{~J}$. Orear, "Least-squares when both variables have uncertainties," Am. J. Phys. 50, 912-916 (1982).

${ }^{5} \mathrm{M}$. Lybanon, "A better least-squares method when both variables have uncertainties," Am. J. Phys. 52, 22-26 and 276-278 (1984).

"S. D. Christian and E. E. Tucker, "Linear least squares analysis with dependent and independent variables subject to error," Am. Lab. 18(2), 33-36 (1986).

${ }^{7}$ This derivation is due to Dr. M. Lybanon, to whom I am grateful for permission to reproduce it.

"J. H. Williamson, "Least-squares fitting of a straight line," Can. J. Phys. 46, 1845-1847 (1968).

${ }^{9}$ W. E. Deming, Statistical Adjustment of Data (Dover, New York, 1964). See in particular Sec. 20. Eq. (21) makes the usual assumption that the errors in $x$ and $y$ are uncorrelated.

${ }^{10} \mathrm{P}$. R. Bevington, Data Reduction and Error Analysis for the Physical Sciences (McGraw-Hill, New York, 1969), Chap. 6.

"W. Jefferys, "On the method of least squares," Astron. J. 85, 177-181 (1980).

${ }^{12}$ D. R. Powell and J. R. McDonald, "A rapidly converging iterative method for the solution of the generalized nonlinear least squares problem," Comput. J. 15, 148-156 (1972).

${ }^{13} \mathrm{~W}$. H. Southwell, "Fitting data to nonlinear functions with uncertainties in all measurement variables," Comput. J. 19, 69-73 (1976).

${ }^{14} \mathrm{~K}$. Pearson, "On lines and planes of closest fit to systems of points in space," Philos. Mag. 2, 559-572, 6th series (1901).

${ }^{15}$ Since the data in Table I are given to only two significant figures, the number of digits quoted here for $\sigma_{m}, \sigma_{c}$, and $S$ are not, strictly speaking, justified. The point is to provide figures against which others can compare the results of their own algorithms. If the data in Table I are regarded as exact (that is, as being followed by an infinite number of zeros $)$, then one finds $(m, c)=(-0.48053341,5.47991022)$, $S=11.86635319,\left(\sigma_{m}, \sigma_{c}\right)_{\text {cho }}=(0.07017175,0.35554746)$, and $\left(\sigma_{m}, \sigma_{c}\right)_{\text {calc }}=(0.07062027,0.35924652)$.

I'D. P. Chong, private communication (1990).

${ }^{17} \mathrm{~W}$. Jefferys, private communication (1990). 